\title{
Development of the Adomian's Method for Solving non-linear Fredholm-Fredholm Integral Equations
}

\author{
Junaid Idrees Mustafa \\ Department of Mathematics / College of Education \\ University of Mosul
}

Received
$30 / 09 / 2009$

Accepted

30 / 09 / $2009 \quad 05 / 01 / 2010$

\begin{abstract}
(الملخص:
اقترحت طريقة جديدة مباشرة ومقبولة لحساب حل عددي للمعادلة التكاملية غير الخطية

من نوع فريد هولم -فريدهولم.

أساس الطريقة هي حل متسلسلة لكمية غير معرفة بطريقة ادمون. حصلنا على نتائج

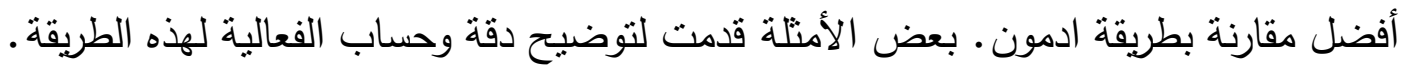

\begin{abstract}
:
A new and effective direct method to determine the numerical solution of non- linear Fredholm-Fredholm integral equations is proposed.

The method is based on a series solution for the unknown quantity by Adomian's method. We obtained very good results compared with classic Adomian's method Some numerical examples are provided to illustrate accuracy and computational efficiency of the method.
\end{abstract}

\section{Introduction}

Over several decades, numerical method in electromagnetic have been the subject of extensive researches. many problems in electromagnetic can be modeled by integral and integro-differential equation for example, electric field integral equation (EFIE) and magnetic field integral equation (MFIE)[3]. Some papers studied the problem of existence and uniqueness of solution of non-linear Volterra-Fredholm integral equations see $[1,6,7]$. Some authors use Adomian's method for solving this equations[4].

In this article we present Adomian's method solution solving nonlinear integral equation of Fredholm-Fredholm type:- 
$\phi(x)=f(x)+\int_{a}^{b} k_{1}(x, t) g_{1}(\phi(t)) d t+\int_{a}^{b} k_{2}(x, t) g_{2}(\phi(t)) d t \quad x, t \in[a, b]$

where

$$
g_{1}(\phi(x))=\phi^{p_{1}}(x)
$$

$$
g_{2}(\phi(x))=\phi^{p_{2}}(x)
$$

$p_{1} \neq p_{2} \geq 2$ are positive integers, $k_{1}(x, t)$ and $k_{2}(x, t)$ are referred to as the kernel and $f(x)$ a given function, $g, k$ and $f$ are non functions.

\section{Analysis:}

In this section, we first describe the algorithm of Adomian's method as it applies to a general non-linear equation of the form[2]:

$\phi=f+N(\phi)$

or

$\phi-N(\phi)=f$

where $\quad N$ is a non-linear operator on a Hilbert space $\mathrm{H}$ and $f$ is a known element of $\mathrm{H}$. we assume that for a given $f$.

It is well known that (AM) considers $\phi(x)$ as an infinite sum of components $\phi_{n}(x)$ defined by:

$\phi(x)=\sum_{n=0}^{\infty} \phi_{n}(x)=\phi_{o}+\phi_{1}+\phi_{2}+\phi_{3}+\ldots$

and the non-linear operator $N$ can be decomposed into $A_{o}\left(\phi_{o}\right)+A_{1}\left(\phi_{o}, \phi_{1}\right)+A_{2}\left(\phi_{o}, \phi_{1}, \phi_{2}\right)+A_{3}\left(\phi_{o}, \phi_{1}, \phi_{2}, \phi_{3}\right)+$

that is :

$$
N(\phi)=\sum_{n=0}^{\infty} A_{n}\left(\phi_{O}, \phi_{1}, \phi_{2}, . . \phi_{n}\right)
$$

where $A_{n}$ are the so-called Adomian polynomial's.

Substituting (2.3) and (2.5) into the functional equation (2.2) yields:

$\sum_{n=0}^{\infty} \phi_{n}(x)-\sum_{n=0}^{\infty} A_{n}=f$

If the series in (2.6) is convergent, then (2.6) holds upon setting:

$$
\begin{aligned}
& \quad \phi_{o}=f \\
& \phi_{1}=A_{o}\left(\phi_{o}\right) \\
& \phi_{2}=A_{1}\left(\phi_{o}, \phi_{1}\right) \\
& \vdots \\
& \phi_{n}=A_{n-1}\left(\phi_{o}, \phi_{1}, \phi_{2}, \ldots \ldots . \phi_{n-1}\right)
\end{aligned}
$$


Thus, one can recursively determine every term of the series $\sum_{n=o}^{\infty} \phi_{n}$. The convergence of this series has been established. The two hypotheses necessary for proving convergence of the Adomian's method as given in [5] are as follows.

Condition 2.1: The non- linear function equation (2.2) has a series solution $\sum_{n=o}^{\infty} \phi_{n}$ such that $\sum_{n=o}^{\infty}(1+\epsilon)^{n}\left|\phi_{n}\right|<\infty$. Where $\in>o$ may be very small.

Condition (2.2): The non-linear operator $N(\phi)$ can be developed in series $N(\phi)=\sum_{n=o}^{\infty} \alpha_{n} \phi^{n}$.

These hypotheses, for proving convergence, are generally satisfied in physical problems [5].

The modified Adomian method [4] may be roughly described as a reassignment of the initial approximates $\phi_{o}$ and $\phi_{1}$. In particular, if $f$ is split into two functions, say, $f=f 1+f 2$, then we may rewrite (2.7) as:

$$
\begin{aligned}
& \phi_{o}=f 1 \\
& \phi_{1}=f 2+A_{o}\left(\phi_{o}\right) \\
& \phi_{2}=A_{1}\left(\phi_{o}, \phi_{1}\right) \\
& \vdots \\
& \phi_{n}=A_{n-1}\left(\phi_{o}, \phi_{1}, \phi_{2}, \ldots \ldots, \phi_{n-1}\right)
\end{aligned}
$$

The choice of how to assign $\phi_{O}$ and $\phi_{1}$ is experimental, yet it leads to less computational and does accelerate the convergence.

To compute Adomian polynomials we as a new method mentioned in [4]. Consider the equation (1.1), to solve (1.1) by AM, we write

$$
\phi(x)=\sum_{n=0}^{\infty} \phi_{n}(x)
$$

and

$$
\begin{aligned}
& \phi^{p 1}(x)=\sum_{n=o}^{\infty} A_{n}\left(\phi_{o}, \phi_{1}, \phi_{2}, \ldots . .\right) \quad p_{1} \neq p_{2} \geq 2 \\
& \phi^{p 2}(x)=\sum_{n=o}^{\infty} A_{n}\left(\phi_{o}, \phi_{1}, \phi_{2}, \ldots . .\right)
\end{aligned}
$$


and substituted the series (2.3), (2.9) and (2.10) in to (1.1) giving

$$
\begin{gathered}
\sum_{n=o}^{\infty} \phi_{n}(x)=f(x)+\int_{a}^{b} K_{1}(x, t)\left(\sum A_{n}\left(\phi_{o}(t), \phi_{1}(t), \ldots \ldots . \phi_{n}(t)\right)\right) d t+ \\
\int_{a}^{b} K_{2}(x, t)\left(\sum A_{n}\left(\phi_{o}(t), \phi_{1}(t), \ldots \ldots \phi_{n}(t)\right)\right) d t
\end{gathered}
$$

or

$$
\begin{gathered}
\phi_{O}+\phi_{1}+\phi_{2}+\phi_{3}+\ldots=f(x)+\int_{a}^{b} K_{1}(x, t)\left(A_{O}+A_{1}+\ldots \ldots . .\right) d t+ \\
\int_{a}^{b} K_{2}(x, t)\left(A_{O}+A_{1}+\ldots \ldots . .\right) d t
\end{gathered}
$$

If the series is convergent, then we can determined each term of the series $\sum_{n=o}^{\infty} \phi_{n}(x)$ recursively:

$\phi_{o}=f(x)$

$\phi_{1}=\int_{a}^{b} K_{1}(x, t) A_{o}\left(\phi_{o}\right) d t+\int_{a}^{b} K_{2}(x, t) A_{o}\left(\phi_{o}\right) d t$

$\phi_{2}=\int_{a}^{b} K_{1}(x, t) A_{1}\left(\phi_{o}, \phi_{1}\right) d t+\int_{a}^{b} K_{2}(x, t) A_{1}\left(\phi_{o}, \phi_{1}\right) d t$

$\phi_{n}=\int_{a}^{b} K_{1}(x, t) A_{n-1}\left(\phi_{o}, \ldots \ldots . \phi_{n-1}\right) d t+\int_{a}^{b} K_{2}(x, t) A_{n-1}\left(\phi_{o}, \ldots \ldots \phi_{n-1}\right) d t$

The algorithm in (2.13) determines the $\phi_{n}$ 's and hence the solution $\phi$ can be determined by (2.3). we will also apply the modified decomposition by writing $f=f 1+f 2$ with appropriate choice for $\phi_{o}$ and $\phi_{1}$.

Then the Adomian polynomial $A_{o}$ depends up on $\phi_{o}$ with order 0 , $A_{1}$ depends up on $\phi_{o}$ and $\phi_{1}$ with order 1 and so forth, see[4], we will have $A_{n}$ as follows: 


$$
\begin{aligned}
& A_{o}=g\left(\phi_{O}\right) \\
& A_{1}=\phi_{1} g^{(1)}\left(\phi_{o}\right) \\
& A_{2}=\phi_{2} g^{(1)}\left(\phi_{o}\right)+\frac{\phi_{1}^{2}}{2 !} g^{(2)}\left(\phi_{o}\right) \\
& A_{3}=\phi_{3} g^{(1)}\left(\phi_{o}\right)+\phi_{1} \phi_{2} g^{(2)}\left(\phi_{o}\right)+\frac{\phi_{1}^{3}}{3 !} g^{(3)}\left(\phi_{o}\right) \\
& A_{4}=\phi_{4} g^{(1)}\left(\phi_{o}\right)+\phi_{1} \phi_{3} g^{(2)}\left(\phi_{o}\right)+\frac{\phi_{2}^{2}}{2 !} g^{(2)}\left(\phi_{o}\right)+\frac{\phi_{1}^{2}}{2} \phi_{2} g^{(3)}\left(\phi_{o}\right) \\
& A_{5}=\phi_{5} g^{(1)}\left(\phi_{o}\right)+\phi_{1} \phi_{4} g^{(2)}\left(\phi_{o}\right)+\phi_{2} \phi_{3} g^{(2)}\left(\phi_{o}\right)+\frac{\phi_{1}^{2}}{2} \phi_{3} g^{(3)}\left(\phi_{o}\right) \\
& +\frac{1}{2} \phi_{2}^{2} \phi_{1} g^{(3)}\left(\phi_{o}\right) \\
& A_{6}=\phi_{6} g^{(1)}\left(\phi_{o}\right)+\phi_{1} \phi_{5} g^{(2)}\left(\phi_{o}\right)+\phi_{2} \phi_{4} g^{(2)}\left(\phi_{o}\right)+\phi_{1} \phi_{2} \phi_{3} g^{(3)}\left(\phi_{o}\right) \\
& +\frac{\phi_{3}^{2}}{2} g^{(2)}\left(\phi_{o}\right)+\frac{\phi_{1}^{2}}{2} \phi_{4} g^{(3)}\left(\phi_{o}\right)+\frac{\phi_{2}^{3}}{3 !} g^{(3)}\left(\phi_{o}\right) \\
& A_{7}=\phi_{7} g^{(1)}\left(\phi_{o}\right)+\phi_{1} \phi_{6} g^{(2)}\left(\phi_{o}\right)+\phi_{2} \phi_{5} g^{(2)}\left(\phi_{o}\right)+\phi_{3} \phi_{4} g^{(2)}\left(\phi_{o}\right)+\frac{\phi_{3}^{2}}{2} \phi_{1} g^{(3)}\left(\phi_{o}\right) \\
& +\frac{\phi_{1}^{2}}{2} \phi_{5} g^{(3)}\left(\phi_{o}\right)+\frac{\phi_{2}^{2}}{2} \phi_{3} g^{(3)}\left(\phi_{o}\right)
\end{aligned}
$$

Go on this course, we will get $A_{n}$

\section{Numerical Examples:}

Here we list the results of approximating some problems solved by Adomian's method and modified Adomian's method.

Example 1: we apply the standard Adomian's method

$$
\phi(x)=f(x)+\int_{0}^{1} K_{1}(x, t) g_{1}(\phi(t)) d t+\int_{0}^{1} K_{2}(x, t) g_{2}(\phi(t)) d t \quad x, t \in[0,1]
$$

where

$$
\begin{aligned}
& f(x)=x^{2}-0.023809 x-0.14 \\
& \phi(x)=x^{2} \\
& K_{1}(x, t)=0.7 \\
& g_{1}(\phi(x))=\phi^{2}(x) \\
& K_{2}(x, t)=x / 6 \\
& g_{2}(\phi(x))=\phi^{3}(x)
\end{aligned}
$$


and solved by two methods Adomian's and modification method, and the results will be compared.

\section{A) classic Adomian's method}

In this example, the non-linear term is $g_{1}(\phi(x))$ and $g_{2}(\phi(x))$ using the algorithm $\phi_{o}(x)=f(x)=x^{2}-0.023809 x-0.14$, then

$$
\begin{aligned}
& \phi_{1}(x)=\int_{0}^{1}(0.7) \phi_{o}^{2}(t) d t+\int_{0}^{1}(x / 6) \phi_{o}^{3}(t) d t \\
& \phi_{2}(x)=\int_{0}^{1}(0.7) 2 \phi_{o}(t) \phi_{1}(t) d t+\int_{0}^{1}(x / 6) 3 \phi_{o}^{2}(t) \phi_{1}(t) d t \\
& \phi_{3}(x)=\int_{0}^{1}(0.7)\left[2 \phi_{o}(t) \phi_{2}(t)+\phi_{1}^{2}(t)\right] d t+\int_{0}^{1}(x / 6)\left[3 \phi_{o}^{2}(t) \phi_{2}(t)+3 \phi_{o}(t) \phi_{1}^{2}(t)\right] d t \\
& \phi_{4}(x)=\int_{0}^{1}(0.7)\left[2 \phi_{o}(t) \phi_{3}(t)+2 \phi_{1}(t) \phi_{2}(t)\right] d t+ \\
& \int_{0}^{1}(x / 6)\left[3 \phi_{o}^{2}(t) \phi_{3}(t)+6 \phi_{o} \phi_{1}(t) \phi_{2}(t)+\phi_{1}^{3}\right] d t \\
& \phi_{5}(x)=\int_{0}^{1}(0.7)\left[2 \phi_{o}(t) \phi_{4}(t)+2 \phi_{1}(t) \phi_{3}(t)+\phi_{2}^{2}(t)\right] d t \quad+ \\
& \int^{1}(x / 6)\left[3 \phi_{o}^{2}(t) \phi_{4}(t)+6 \phi_{o}(t) \phi_{1}(t) \phi_{3}(t)+3 \phi_{o}(t) \phi_{2}{ }^{2}(t)+3 \phi_{1}{ }^{2}(t) \phi_{2}(t)\right] d t \\
& 0 \\
& \phi_{6}(x)=\int_{0}^{1}(0.7)\left[2 \phi_{o}(t) \phi_{5}(t)+2 \phi_{2}(t) \phi_{3}(t)+2 \phi_{1}(t) \phi_{4}(t)\right] d t+ \\
& \int_{0}^{1}(x / 6)\left[3 \phi_{o}^{2}(t) \phi_{5}(t)+6 \phi_{o}(t) \phi_{2}(t) \phi_{3}(t)+6 \phi_{o}(t) \phi_{1}(t) \phi_{4}(t)+\right. \\
& \left.3 \phi_{1}^{2}(t) \phi_{3}(t)+3 \phi_{2}^{2}(t) \phi_{1}(t)\right] d t
\end{aligned}
$$




$$
\begin{aligned}
& \phi_{7}(x)=\int_{0}^{1}(0.7)\left[2 \phi_{o}(t) \phi_{6}(t)+2 \phi_{2}(t) \phi_{4}(t)+2 \phi_{1}(t) \phi_{5}(t)+\phi_{3}^{2}(t)\right] d t+ \\
& \int_{0}^{1}(x / 6)\left[3 \phi_{o}^{2}(t) \phi_{6}(t)+6 \phi_{o}(t) \phi_{2}(t) \phi_{4}(t)+6 \phi_{o}(t) \phi_{1}(t) \phi_{5}(t)+\right. \\
& \phi_{8}(x)=\int_{0}^{1}(0.7)\left[2 \phi_{o}(t) \phi_{7}(t)+2 \phi_{1}(t) \phi_{6}(t)+2 \phi_{2}(t) \phi_{5}(t)+2 \phi_{3}(t) \phi_{4}(t)\right] d t+ \\
& \int_{0}^{1}(x / 6)\left[3 \phi_{o}^{2}(t) \phi_{7}(t)+6 \phi_{o}(t) \phi_{1}(t) \phi_{6}(t)+6 \phi_{0}(t) \phi_{2}(t) \phi_{5}(t)+6 \phi_{o}(t) \phi_{3}(t) \phi_{4}(t)+\right. \\
& \left.3 \phi_{1}(t) \phi_{3}^{2}(t)+3 \phi_{1}^{2}(t) \phi_{5}(t)+3 \phi_{2}^{2}(t) \phi_{3}(t)\right] d t
\end{aligned}
$$

$\phi(x)$ is approximated by using eight terms of Adomian's method $\phi(x) \cong \phi_{o}(x)+\phi_{1}(x)+\phi_{2}(x)+\ldots$

Figure (1.1) shows the approximate solution, it is obvious from that the approximation is good.

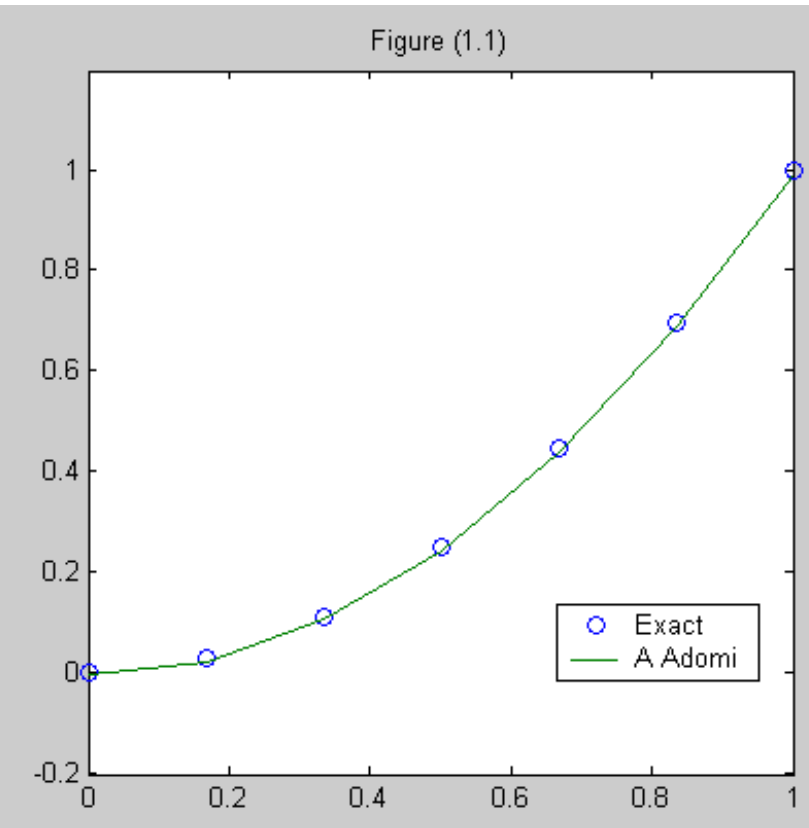

Figure (1.1) comparison of convergence rate for classic Adomian's method and exact solution $x^{2}$ 


\section{B) modified Adomian's method}

assume that $f=f 1+f 2$

then

$$
f 1(x)=x^{2}-0.023809 x \quad f 2(x)=-0.14
$$

let

$\phi_{o}(x)=f 1=x^{2}-0.023809 x \quad$ then

$\phi_{1}(x)=f 2+\int_{0}^{1}(0.7) \phi_{o}{ }^{2}(t) d t+\int_{0}^{1}(x / 6) \phi_{o}{ }^{3}(t) d t$

$\phi_{2}(x)=\int_{0}^{1}(0.7) 2 \phi_{o}(t) \phi_{1}(t) d t+\int_{0}^{1}(x / 6) 3 \phi_{o}{ }^{2}(t) \phi_{1}(t) d t$

We can see also from Figure (1.2) that this modified is very good.

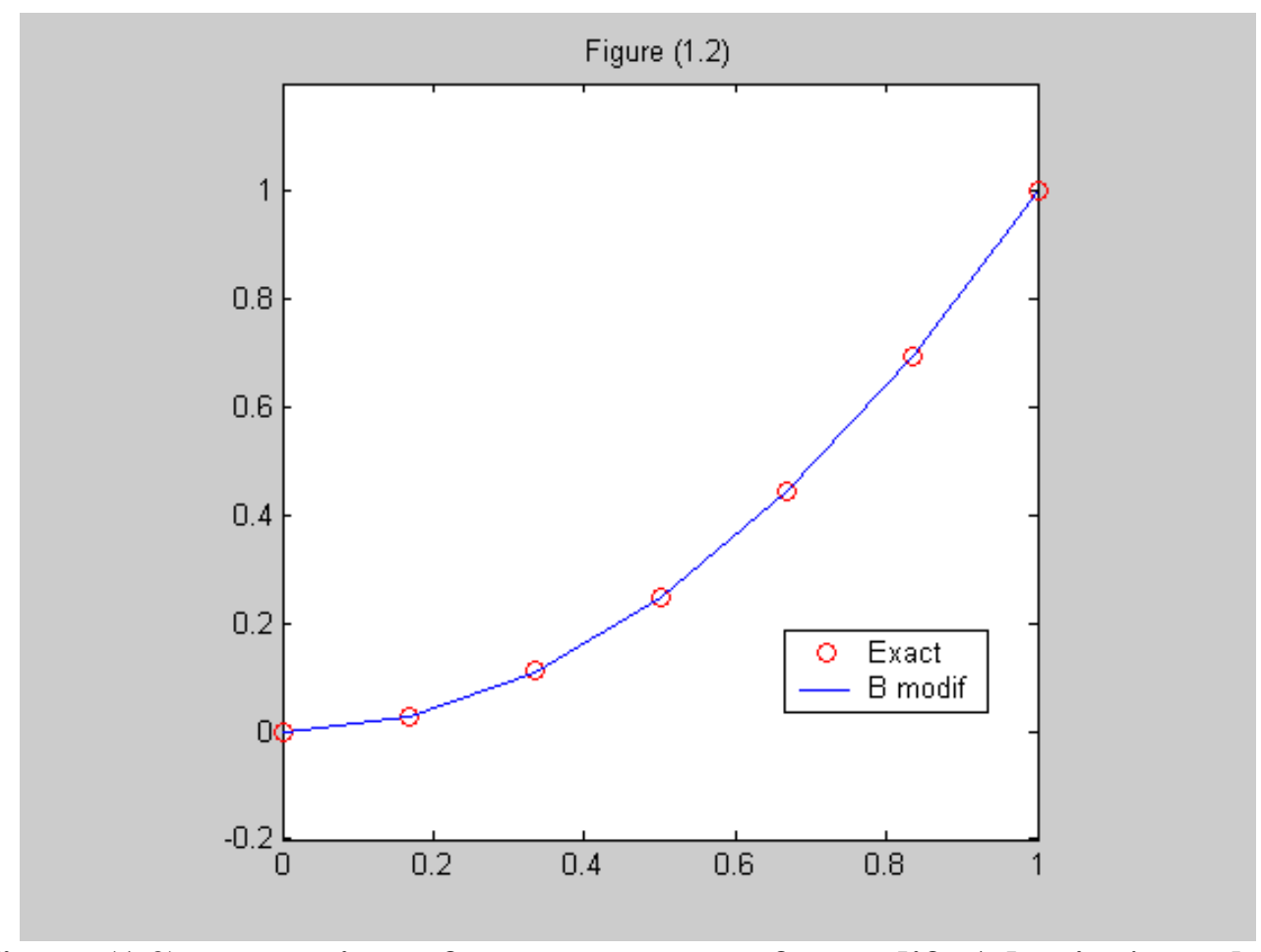

Figure (1.2): comparison of convergence rate for modify Adomian's method and exact solution $x^{2}$.This method was applied at the $6^{\text {th }}$ iteration.

A comparison of the approximate solution between classic and the modified Adomian's method with the exact solution $\phi(x)=x^{2}$ of the integral equation at $x=0,1 / 6,2 / 6,3 / 6,4 / 6,5 / 6$ and 1 yield the errors displayed in table (1.1). 
Table(1.1)

\begin{tabular}{|c|c|c|c|c|c|c|c|}
\hline $\mathrm{x}$ & 0 & 0.16667 & 0.33333 & 0.5 & 0.66667 & 0.83333 & 1 \\
\hline Error by classic & 0.0067 & 0.0069 & 0.0072 & 0.0074 & 0.0077 & 0.0079 & 0.0082 \\
\hline Error by MA & $4.468 \mathrm{e}-4$ & $4.634 \mathrm{e}-4$ & $4.8 \mathrm{e}-4$ & $4.966 \mathrm{e}-4$ & $5.132 \mathrm{e}-4$ & $5.298 \mathrm{e}-4$ & $5.464 \mathrm{e}-4$ \\
\hline
\end{tabular}

\section{Example 2:}

We apply the standard Adomian's method

$$
\phi(x)=f(x)+\int_{0}^{1} K_{1}(x, t) g_{1}(\phi(t)) d t+\int_{0}^{1} K_{2}(x, t) g_{2}(\phi(t)) d t \quad x \in[0,1]
$$

where

$$
\begin{array}{lc}
f(x)=x^{3}-0.076923 x-0.00909 & \phi(x)=x^{3} \\
K_{1}(x, t)=0.1 t & K_{2}(x, t)=x \\
g_{1}(\phi(x))=\phi^{3}(x) & g_{2}(\phi(x))=\phi^{4}(x)
\end{array}
$$

A) classic Adomian's method

$$
\begin{aligned}
& \phi_{O}(x)=x^{3}-0.076923 x-0.00909 \\
& \phi_{1}(x)=\int_{0}^{1}(0.1 t) \phi_{O}^{3}(t) d t+\int_{0}^{1} x \phi_{O}^{4}(t) d t \\
& \phi_{2}(x)=\int_{0}^{1}(0.1 t) 3 \phi_{O}^{2}(t) \phi_{1}(t) d t+\int_{0}^{1} x 4 \phi_{O}^{3}(t) \phi_{1}(t) d t
\end{aligned}
$$

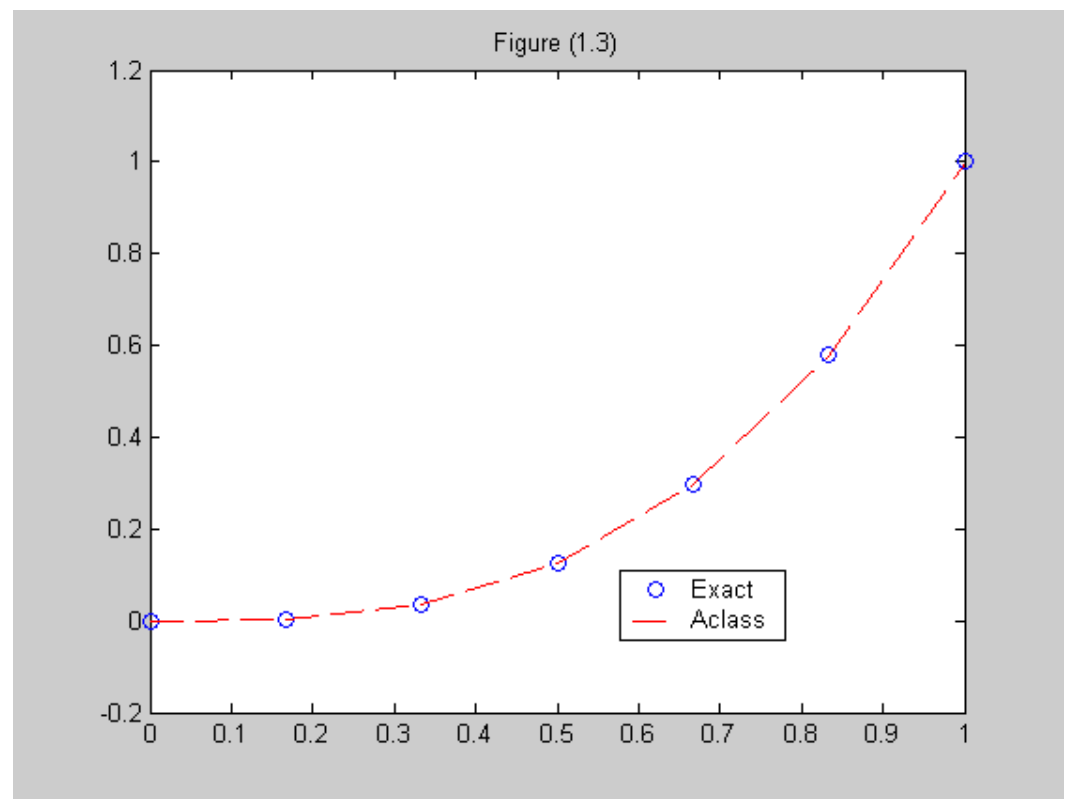

Figure (1.3): classic Adomian's method versus exact solution for $\phi(x)=x^{3}$ 
B) modified Adomian's method assume that

$$
f=f 1+f 2
$$

$$
\text { then } \quad f 1=x^{3}-0.076923 x \quad f 2=-0.00909
$$

Let

$$
\begin{aligned}
& \phi_{O}=f 1=x^{3}-0.076923 x \\
& \phi_{1}(x)=f 2+\int_{0}^{1}(0.1 t) \phi_{O}{ }^{3}(t) d t+\int_{0}^{1} x \phi_{O}{ }^{4}(t) d t \\
& \phi_{2}(x)=\int_{0}^{1}(0.1 t) 3 \phi_{O}{ }^{2}(t) \phi_{1}(t) d t+\int_{0}^{1} x 4 \phi_{O}{ }^{3}(t) \phi_{1}(t) d t
\end{aligned}
$$

Figure (1.4)

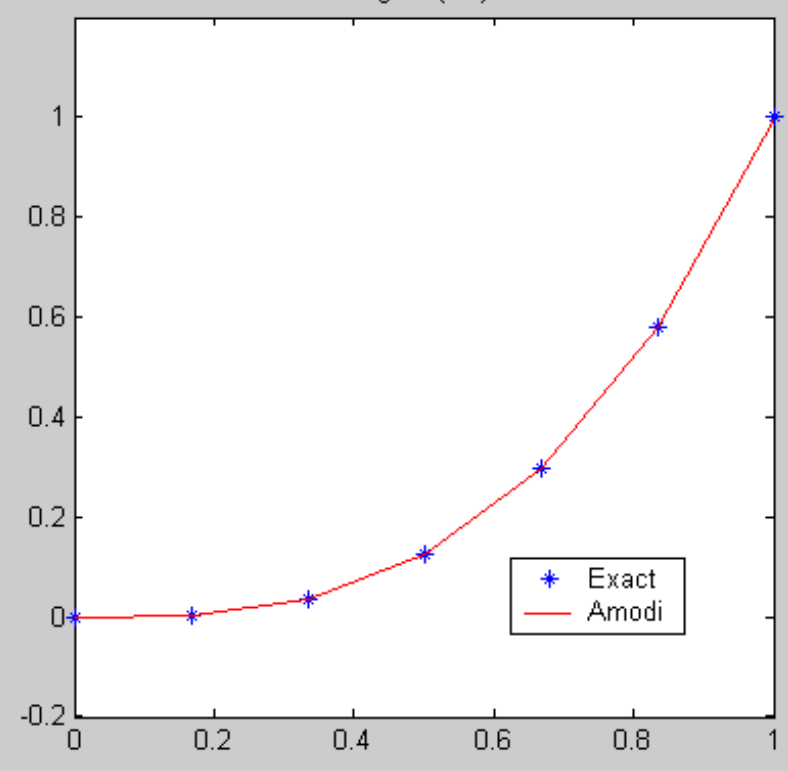

Figure (1.4): classic modify Adomian's method versus exact solution for $\phi(x)=x^{3}$

A comparison of the approximate solution between classic and the modified Adomian's method with the exact solution $\phi(x)=x^{3}$ of the integral equation at $x=0,1 / 6,2 / 6,3 / 6,4 / 6,5 / 6$ and 1 yield the errors displayed in table (1.2).

Table (1.2)

\begin{tabular}{|c|c|c|c|c|c|c|c|}
\hline $\mathrm{x}$ & 0 & 0.16667 & 0.33333 & 0.5 & 0.66667 & 0.83333 & 1 \\
\hline Error by classic & 0.0001 & 0.0003 & 0.0005 & 0.0006 & 0.0008 & 0.0010 & 0.0012 \\
\hline Error by AM & $5.74 \mathrm{e}-4$ & $2.059 \mathrm{e}-4$ & $3.545 \mathrm{e}-4$ & $5.031 \mathrm{e}-4$ & $6.517 \mathrm{e}-4$ & $8.003 \mathrm{e}-4$ & $9.489 \mathrm{e}-4$ \\
\hline
\end{tabular}




\section{Example :3}

Consider the problem

$$
\begin{array}{lc}
\phi(x)=f(x)+\int_{0}^{1} K_{1}(x, t) g_{1}(\phi(t)) d t+\int_{0}^{1} K_{2}(x, t) g_{2}(\phi(t)) d t & x, t \in[0,1] \\
f(x)=x^{4}-0.047619 x-0.071429 & \phi(x)=x^{4} \\
K_{1}(x, t)=t & K_{2}(x, t)=x^{2} \\
g_{1}(\phi(x))=\phi^{3}(x) & g_{2}(\phi(x))=\phi^{5}(x)
\end{array}
$$

A) by classic Adomian's method

$$
\begin{aligned}
& \phi_{O}(x)=x^{2}-0.047619 x-0.071429 \\
& \phi_{1}(x)=\int_{0}^{1} t \phi_{O}{ }^{3}(t) d t+\int_{0}^{1} x^{2} \phi_{O}{ }^{5}(t) d t \\
& \phi_{2}(x)=\int_{0}^{1} t 3 \phi_{O}{ }^{2}(t) \phi_{1}(t) d t+\int_{0}^{1} x^{2} 5 \phi_{O}{ }^{4}(t) \phi_{1}(t) d t \\
& \vdots
\end{aligned}
$$

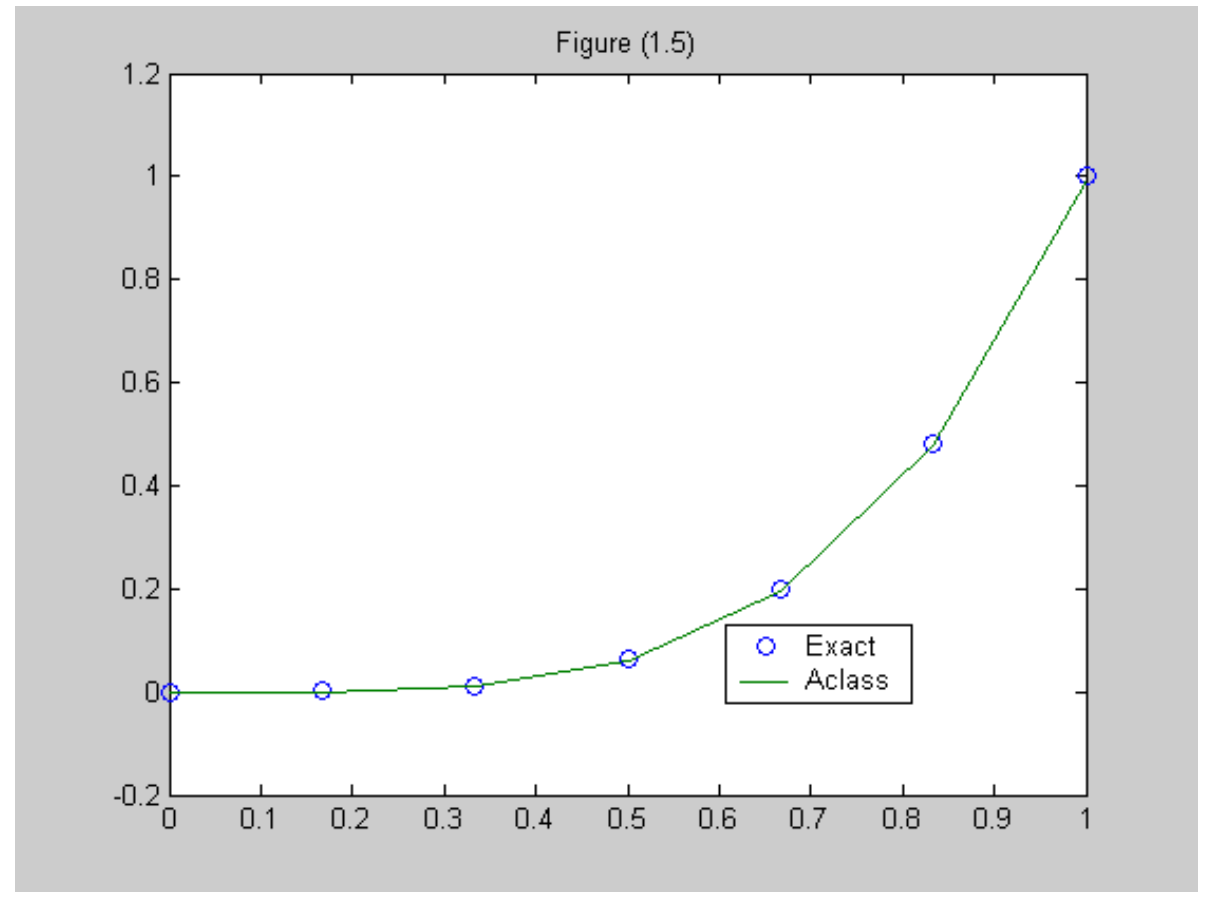

Figure(1.5) comparison of convergence rate for modify Adomian's method and exact solution $\phi(x)=x^{4}$. 
B) by modification method assume that :

$$
f=f 1+f 2
$$

$$
\begin{array}{cl}
\text { then } & f 1=x^{4}-0.047619 x \quad f 2=-0.071429 \\
\text { let } & \phi_{o}=f 1=x^{4}-0.047619 x
\end{array}
$$

then

$$
\begin{aligned}
& \phi_{1}(x)=f 2+\int_{0}^{1} t \phi_{o}^{3}(t) d t+\int_{0}^{1} x^{2} \phi_{o}{ }^{5}(t) d t \\
& \phi_{2}(x)=\int_{0}^{1} t 3 \phi_{o}^{2}(t) \phi_{1}(t) d t+\int_{0}^{1} x^{2} 5 \phi_{o}^{4}(t) \phi_{1}(t) d t
\end{aligned}
$$

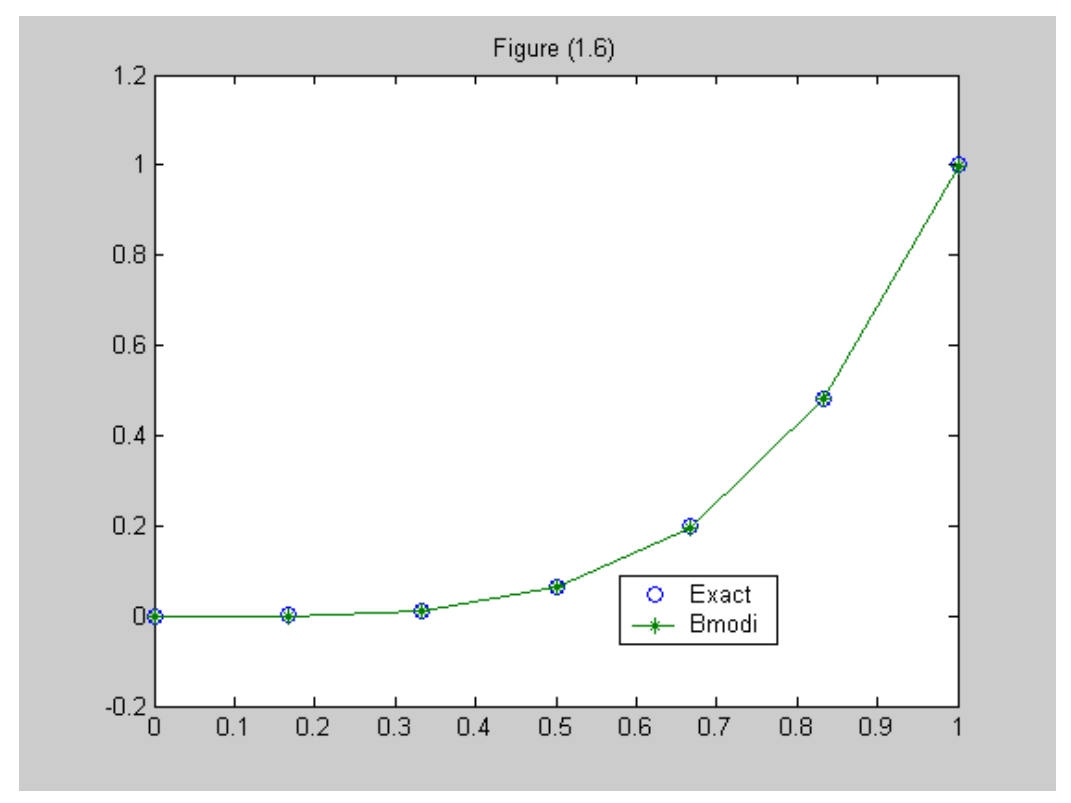

Figure (1.6): comparison of convergence rate for modify Adomian's method and exact solution $\phi(x)=x^{4}$. This method was applied at the $6^{\text {th }}$ iteration.

A comparison of the approximate solution between classic and the modified Adomian's method with the exact solution $\phi(x)=x^{4}$ of the integral equation at $x=0,1 / 6,2 / 6,3 / 6,4 / 6,5 / 6$ and 1 yield the errors displayed in table (1.3).

Table (1.3)

\begin{tabular}{|c|c|c|c|c|c|c|c|}
\hline $\mathrm{X}$ & 0 & 0.16667 & 0.33333 & 0.5 & 0.66667 & 0.83333 & 1 \\
\hline Error by classic & 0.003 & 0.0031 & 0.0033 & 0.0038 & 0.0045 & 0.0035 & 0.0064 \\
\hline Error by AM & $3.656 \mathrm{e}-4$ & $3.744 \mathrm{e}-4$ & $4.010 \mathrm{e}-4$ & $4.453 \mathrm{e}-4$ & $5.073 \mathrm{e}-4$ & $5.87 \mathrm{e}-4$ & $6.845 \mathrm{e}-4$ \\
\hline
\end{tabular}




\section{Conclusion:}

The Adomian's and modified Adomian's method are applied to solve the Fredholm-Fredholm integral equations. The method is based upon changing the non- linear operator in to finite series. Hence this Development for Adomian's is much faster than classic Adomian's method and keeping the accuracy of the solution. The numerical examples support this claim.

\section{References:}

1) A. A. El. Bary, Solution of Fredholm-Volerra Integral Equation of the Second kind in Series Form 4(4), 537-538, 2004.

2) A. Ranjbra, J. Biazar, A Comparison Between Newton's Method and A.D.M for solving Special Fredholm Integral Equations. No.5,215-222,2007.

3) E. Babolian, Z. Masouri, S. Hatamzadeh, New Direct Method to Solve non- linear Volterra-Fredholm Integral and Integrodifferential Equations using Operational Matrix with Block-pulse Functions, vol. 8, 59 -76, 2008.

4) E. Deeba and S. xie, Numerical Approximation for Integral Equations vol.20, 1057-1065, 2003.

5) G. Adomian, Solving Frontier Problems of Physic: the Decomposition Method, Fundamental Theories of Physics, vol. 60, Kluwer Academic Publishers Group, Dordrecht, 1994.

6) Haribhau L. Tidke, Existence of Globel Solutions to non-linear Mixed Volterra-Fredholm Integrodifferential Equations with Nonlocal Conditions, vol.55, 1-7, 2009.

7) K. Balach and P. prakash, Exastence of Solutions of Non-linear Fuzzy Volterra-Fredholm integral equations 33(3), 329-343, 2002. 